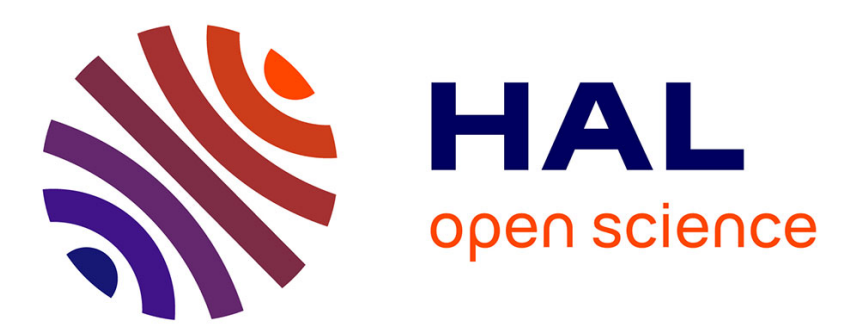

\title{
Application of Whitney elements for the reconstruction of electric arc current density in low-voltage circuit breakers
}

\author{
Jinlong Dong, Luca Di Rienzo, Olivier Chadebec, Jianhua Wang
}

\section{To cite this version:}

Jinlong Dong, Luca Di Rienzo, Olivier Chadebec, Jianhua Wang. Application of Whitney elements for the reconstruction of electric arc current density in low-voltage circuit breakers. COMPEL: The International Journal for Computation and Mathematics in Electrical and Electronic Engineering, 2019, 38 (3), pp.1036-1047. 10.1108/COMPEL-09-2018-0359 . hal-02995399

\section{HAL Id: hal-02995399 \\ https://hal.science/hal-02995399}

Submitted on 9 Nov 2020

HAL is a multi-disciplinary open access archive for the deposit and dissemination of scientific research documents, whether they are published or not. The documents may come from teaching and research institutions in France or abroad, or from public or private research centers.
L'archive ouverte pluridisciplinaire HAL, est destinée au dépôt et à la diffusion de documents scientifiques de niveau recherche, publiés ou non, émanant des établissements d'enseignement et de recherche français ou étrangers, des laboratoires publics ou privés. 


\title{
Application of Whitney elements for the reconstruction of electric arc current density in low-voltage circuit breakers
}

\author{
Jinlong Dong
}

School of Electrical Engineering, State Key Laboratory of Electrical Insulation and Power Equipment, Xi'an Jiaotong University, Xi'an, China and Dipartimento di Elettronica, Informazione e Bioingegneria, Politecnico di Milano, Milano, Italy

\section{Luca Di Rienzo}

Dipartimento di Elettronica, Informazione e Bioingegneria, Politecnico di Milano, Milano, Italy

Olivier Chadebec

Univ. Grenoble Alpes, CNRS, Grenoble INP, G2Elab, Grenoble, France, and

\author{
Jianhua Wang
}

School of Electrical Engineering, State Key Laboratory of Electrical Insulation and Power Equipment, Xi'an Jiaotong University, Xi'an, China

\begin{abstract}
Purpose - This paper aims to present the mathematical formulations of a magnetic inverse problem for the electric arc current density reconstruction in a simplified arc chamber of a low-voltage circuit breaker.

Design/methodology/approach - Considering that electric arc current density is a zero divergence vector field, the inverse problem can be solved in Whitney space $\mathrm{W}^{2}$ in terms of electric current density $\mathbf{J}$ with the zero divergence condition as a constraint or can be solved in Whitney space $\mathrm{W}^{1}$ in terms of electric vector potential $\mathbf{T}$ where the zero divergence condition naturally holds. Moreover, the tree gauging condition is applied to ensure a unique solution when solving for the vector potential in space $\mathrm{W}^{1}$. Tikhonov regularization is used to treat the ill-posedness of the inverse problem complemented with $\mathrm{L}$-curve method for the selection of regularization parameters. A common mode approach is proposed, which solves for the reduced electric vector potential representing the internal current loops instead of solving for the total electric vector potential. The proposed inversion approaches are numerically tested starting from simulated magnetic field values.

Findings - With the common mode approach, the reconstruction of current density is significantly improved for both formulations using face elements in space $\mathrm{W}^{2}$ and using edge elements in space $\mathrm{W}^{1}$. When solving the inverse problem in space $\mathrm{W}^{1}$, the choice of the regularization operator has a key role to obtain a good reconstruction, where the discrete curl operator is a good option. The standard Tikhonov regularization obtains a good reconstruction with $\mathbf{J}$-formulation, but fails in the case of $\mathbf{T}$-formulation. The use of edge elements requires a tree-cotree gauging to ensure the uniqueness of $\mathbf{T}$. Moreover, additional efforts have to be taken to find an optimal regularization operator and an optimal tree when using edge elements. In conclusion, the $\mathbf{J}$-formulation is to be preferred.
\end{abstract}

This work was supported by the National Natural Science Foundation of China under Grant 51477129 and the China Scholarship Council under Grant 201706280142. 
Originality/value - The proposed approaches are able to reconstruct the three-dimensional electric arc current density from its magnetic field in a non-intrusive manner. The formulations enable us to incorporate $a$ priori knowledge of the unknown current density into the solution of the inverse problem, including the zero divergence condition and the boundary conditions. A common mode approach is proposed, which can significantly improve the current density reconstruction.

Keywords Inverse problems, Finite element method, Whitney forms

Paper type Research paper

\section{Introduction}

Low voltage circuit breakers are used to protect people and electrical equipment in power systems by switching off the fault electric current. The current interruption in circuit breakers is characterized by electric arcs. When a fault current occurs in an electrical circuit, an electric arc is ignited during the contacts opening process. Then the electric arc slides between two electrodes due to the electromagnetic force and gas flow; finally, it is extinguished in splitter plates (Yang, 2013; Chadebec et al., 2004). Current density distribution in electric arcs gives us very important information to understand the distribution of temperature, the erosion ratio of electrodes and the interaction of arcs with surroundings. The corresponding magnetic field can be studied under magnetostatic approximation (Wu et al., 2006). The better knowledge of the electric arc current density helps to understand the complex physical behavior of arc plasmas.

At present, available diagnostic techniques to investigate the arc dynamics in a circuit breaker include optical and magnetic methods. Optical diagnostic methods include chargecoupled device (CCD) cameras (Lindmayer et al., 2006), optical fiber arrays (McBride et al., 2010) and spectrum analysis (Hong et al., 2005), whereas the use of the spectrum analysis is limited due to its low temporal resolution. CCD cameras and optical fiber arrays are able to study the arc commutation and movement with high temporal and spatial resolution. However, optical diagnostic techniques require transparent walls or holes drilled on the wall in the experimental device, leading to dramatic effects on the characteristics of the arc plasma (Shea, 2004). Optical methods are, therefore, intrusive and can only obtain the twodimensional optical images of the electric arc in a light pattern, which is not the current density.

On the other hand, magnetic diagnostic techniques are fully non-intrusive methods. In Brdys et al. (2002) and Toumazet et al. (2005), the electrodes and the arc column are simplified to be a succession of two-dimensional linear current lines and the average arc positions are obtained by measuring the magnetic field near the sidewall of the device. In Brdys et al. (2010), the electric arc is modeled as a two-dimensional rectangular current sheet and the current density distribution in the sheet is reconstructed from the magnetic field with a deconvolution approach. However, these models are oversimplified compared with a realistic electric arc and unable to determine the three-dimensional electric arc current density.

This work focuses on a non-intrusive diagnostic technique able to reconstruct the three-dimensional arc current density from its magnetic field by solving a magnetic inverse problem. Different formulations of the magnetic inverse problem are numerically studied in Ghezzi et al. (2012) and Dong et al. (2018). However, a key point for this approach is how to implement the zero divergence condition and the boundary conditions for the reconstructed current density. To this scope, we apply Whitney elements in the formulation of the magnetic inverse problem. The use of Whitney elements in the identification of current distributions is already proposed to model bioelectric current sources in electroencephalography and magnetoencephalography in (Tanzer et al., 2005; 
Pursiainen et al., 2011; Bauer et al., 2015), but no condition on the current density divergence is imposed.

In this work, the magnetic inverse problem is formulated with both face elements in space $\mathrm{W}^{2}$ in terms of electric current density $\mathbf{J}$ and edge elements in space $\mathrm{W}^{1}$ in terms of electric vector potential $\mathbf{T}$. The implementations of the zero divergence condition and the boundary conditions for both formulations are presented. The inverse problem is solved with the help of Tikhonov regularization to address the ill-posedness. Comparisons between $\mathbf{J}$-formulation and $\mathbf{T}$-formulation are carried out. Moreover, a common mode approach is proposed to improve the reconstructions.

\section{Forward and inverse problem formulations}

Governing differential equations

A simplified arc chamber with two parallel electrodes is considered, as in Figure 1. A planar sensor array is used to perform the magnetic measurements. We assume magnetostatic conditions over the whole domain $\Omega$, i.e. the displacement current is neglected due to the low-frequency applications of low voltage circuit breakers. Therefore, the current density is a zero divergence vector field:

$$
\operatorname{div} \mathbf{J}=0,
$$

in the problem's domain $\Omega$, complemented by boundary conditions:

$$
\mathbf{n} \cdot \mathbf{J}=0 \text { on } S_{J}
$$

Figure 1.

Model geometry and setup of the magnetic sensor array

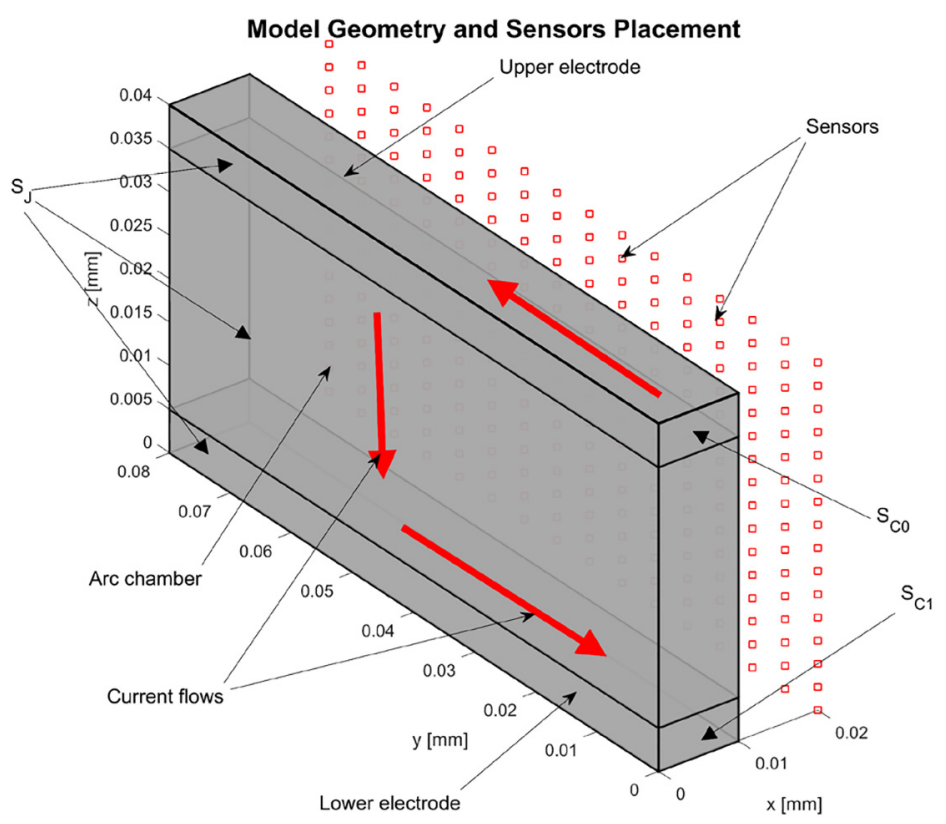




$$
\int_{S_{C i}} \mathbf{n} \cdot \mathbf{J} d S= \pm I_{t o t} \text { on } S_{C i}
$$

on the boundary $\partial \Omega=S_{J} \cup S_{C O} \cup S_{C 1}$. As shown in Figure $1, S_{C 0}$ is the terminal section of the upper electrode where the current flows in and $S_{C 1}$ is the terminal section of the lower electrode where the current flows out. $S_{J}$ is the rest surface of the model with no current flowing out.

In this study, only linear ferromagnetic materials are considered leading to a linear magnetic inverse problem. The corresponding magnetic field equations read:

$$
\begin{gathered}
\operatorname{curl} \mathbf{B}=\mu \mathbf{J}, \\
\operatorname{div} \mathbf{B}=0,
\end{gathered}
$$

In the above equations (1) to (5), $\mathbf{J}$ is the current density, $\mathbf{B}$ the magnetic induction, $\mu$ the permeability and $\mathbf{n}$ the unit normal vector to the boundary. $I_{\text {tot }}$ is the total conduction current.

In this paper, we focus on the solution of the magnetic inverse problem with the incorporation of equations (1) to (3). Thus, we can start from a given reference current density and then calculate the corresponding magnetic field using magnetostatic equations (4) and (5). We then reconstruct the current density from the obtained magnetic field by solving the inverse problem; and finally, compare reconstructions with reference current densities. The modeling of the forward problem considering eddy currents is not in the scope of our work.

\section{Inverse problem formulation using Whitney elements}

The shape function spaces are defined using Whitney elements and $\mathrm{W}^{0}, \mathrm{~W}^{1}, \mathrm{~W}^{2}$ and $\mathrm{W}^{3}$ are the nodal, edge, face and volume function spaces, respectively (Bossavit, 1988). The relations between these spaces are given by De Rham's diagram (Bossavit, 1988):

$$
\mathrm{W}^{0} \stackrel{\operatorname{grad} w_{n}}{\longrightarrow} \mathrm{W}^{1} \stackrel{\operatorname{curl} \mathbf{w}_{\mathrm{e}}}{\longrightarrow} \mathrm{W}^{2} \stackrel{\operatorname{div} \mathbf{w}_{\mathrm{f}}}{\longrightarrow} \mathrm{W}^{3} .
$$

The tetrahedral mesh is used for the discretization of the problem's domain $\Omega$, with $N$ nodes, $E$ edges, $F$ facets and $V$ elements. On a face with nodes $m, n$ and $p$, where its orientation is given by the node order $(m, n, p)$, the face shape function belonging to space $\mathrm{W}^{2}$ can be expressed with nodal shape functions as:

$$
\boldsymbol{w}_{\boldsymbol{f}}=2\left(w_{m} \nabla w_{n} \times \nabla w_{p}+w_{n} \nabla w_{p} \times \nabla w_{m}+w_{p} \nabla w_{m} \times \nabla w_{n}\right) .
$$

Representing the current density $\mathbf{J}$ in $\mathrm{W}^{2}$ using face elements, the forward problem reads:

$$
[B]=[L][J],
$$

where $[B]$ is the vector of magnetic induction, $[J]$ is the vector of the degrees of freedom in $\mathrm{W}^{2}$ and $[L]$ is the kernel matrix. Using Biot-Savart law, the generic element of kernel matrix $[L]$ corresponding to the $i$-th sensor and $j$-th face function $\boldsymbol{w}_{f j}$ is calculated by:

$$
[L]_{i j}=\frac{\boldsymbol{\mu}_{0}}{4 \pi} \int_{\boldsymbol{\Omega}} \boldsymbol{d}_{\boldsymbol{i}} \cdot \frac{\boldsymbol{w}_{f j}\left(\boldsymbol{r}_{\boldsymbol{s}}\right) \times\left(\boldsymbol{r}_{\boldsymbol{i}}-\boldsymbol{r}_{\boldsymbol{s}}\right)}{\left|\boldsymbol{r}_{\boldsymbol{i}}-\boldsymbol{r}_{\boldsymbol{s}}\right|^{3}} d \boldsymbol{\Omega},
$$


where $\boldsymbol{r}_{i}$ is the position of $i$-th sensor, $\boldsymbol{d}_{i}$ the vector of the orientation of $i$-th sensor and $\boldsymbol{r}_{s}$ the integration point. Single component magnetic field data are used for the reconstruction. The impact of more than one magnetic field component could be investigated, as in (Di Rienzo et al., 2005).

The current density $\mathbf{J}$ is expressed as a linear combination of face shape functions:

$$
\mathbf{J}=\sum_{1}^{F} \boldsymbol{w}_{\boldsymbol{f} \boldsymbol{i}}[J]_{i} .
$$

The integral of the face shape function on its own face, i.e. the flux flowing through its corresponding face, is equal to one and is zero on all the other faces. Thus, the $i$-th element of $[J$ is the current flux flowing through its corresponding face (the $i$-th face). Face shape functions have normal continuity, i.e. when passing from one tetrahedron to its neighboring tetrahedron both sharing the face, the normal component of the current density represented in $\mathrm{W}^{2}$ is continuous, whereas the tangential component may have jumps.

The magnetic inverse problem, i.e. reconstructing the current density from the magnetic field, is known to be ill-conditioned. This leads to highly unstable solutions where any arbitrary measurement errors in the magnetic field could cause large perturbations in the solution. Therefore, it is necessary to use regularization methods to make the ill-posed inverse problem more regular to stabilize the problem. To address the ill-posedness, we use Tikhonov regularization to obtain a least-squares solution by solving for $\left[J\right.$ in $\mathrm{W}^{2}$ :

$$
\min _{[J]}\left\{\|[L][J]-[B]\|_{2}^{2}+\lambda^{2}\|[K][J]\|_{2}^{2}\right\},
$$

where $[K]$ is referred as the matrix of the regularization operator and the scalar $\lambda \geq 0$ is the regularization parameter.

Regularization operator $[K]$ and scalar parameter $\lambda$ are essential parameters to ensure a stable and useful solution from the Tikhonov regularized least-squares problem. Many works explore various appropriate options for $[K]$ and $\lambda$ (Hasani Eskandari and Zareamoghaddam, 2014). The regularization operator is related to the prior information about the solution. Particularly, the most common choice for $[K]$ is the identity matrix $[I]$ with the same dimension of the vector of unknowns [J], where the problem defined by equation (11) is reduced as the standard Tikhonov regularization. We use the L-curve method for the choice of a scalar parameter $\lambda$ (Calvetti et al., 2004).

\section{Implementation of zero divergence condition}

In a magneto-quasi-static approximation, the current density is a zero divergence vector field, so that $[D][J=\mathbf{0}$, where $[D]$ is the faces-to-volumes incidence matrix serving as the discrete equivalent of the divergence operator. To implement the zero divergence condition in space $\mathrm{W}^{2}$, we solve a constrained least-squares problem with the free divergence condition as a constraint:

$$
\min _{[J]}\left\{\|[L][J]-[B]\|_{2}^{2}+\lambda^{2}\|[J]\|_{2}^{2}\right\} \text {, s.t. }[D][J]=0 .
$$

Alternatively, we can solve the inverse problem in space $\mathrm{W}^{1}$ by introducing a current vector potential $\mathbf{T}$ with $\mathbf{J}=\operatorname{curl} \mathbf{T}$, where $\mathbf{T}$ can be appropriately represented in space $\mathrm{W}^{1}$ 
using edge elements. We denote the vector of degrees of freedom in space $\mathrm{W}^{1}$ as $[T]$ for $\mathbf{T}$, so that $[J]=[C][T]$, where $[C]$ is the edges-to-faces incidence matrix acting as the discrete equivalent of the curl operator. The generic element of $[T]$ is the circulation along the corresponding edge. The edge shape functions have tangential continuity, i.e. the tangential component of $\mathbf{T}$ is continuous, whereas its normal component may have jumps. One remarkable advantage of the current vector potential approach is that zero divergence condition naturally holds noting the vector calculus identity $\operatorname{div}(\operatorname{curl} \mathbf{T}) \equiv 0$. Moreover, the tree-cotree gauging technique is adopted to ensure the uniqueness of the solution for $\mathbf{T}$ (Meunier, 2008). The regularized least-squares solution by solving for [T] in space $\mathrm{W}^{1}$ is given by:

$$
\min _{[T]}\left\{\|[L][C][T]-[B]\|_{2}^{2}+\lambda^{2}\|[K][T]\|_{2}^{2}\right\} .
$$

\section{Implementation of boundary conditions}

The boundary condition defined in equation (2) implies a zero current flux flowing through surface $S_{5}$. Representing the current density with face elements in space $\mathrm{W}^{2}$, we have:

$$
\left.[J]_{i}\right|_{f_{i} \in S_{J}}=0,
$$

for every face element $f_{i}$ located on $S_{j}$. For terminal sections $S_{C O}$ and $S_{C 1}$, the total current flux is known as implied by equation (3). Thus, for the elements located on $S_{C 0}$ and on $S_{C 1}$, we have:

$$
\sum_{f_{i} \in S_{C 0}}[J]_{i}=\sum_{f_{i} \in S_{C 1}}[J]_{i}=I_{t o t}
$$

From equation (2), we have $\mathbf{n} \times \mathbf{T} \mid S_{J}=0$. We set [T] to be zero for every edge element $e_{i}$ located on $S_{J}$, that is:

$$
\left.[T]_{i}\right|_{e_{i} \in S_{J}}=0 .
$$

In addition, equation (15) implies that for the edge elements located on $S_{C O}$ and on $S_{C 1}$, the circulations of $\mathbf{T}$ along the boundaries of terminal sections $\partial S_{C 0}$ and $\partial S_{C 1}$ are the total current, that is:

$$
\sum_{e_{i} \in \partial S_{C 0}}[T]_{i}=\sum_{e_{i} \in \partial S_{C 1}}[T]_{i}=I_{t o t}
$$

\section{Common mode approach}

In this work, we introduce a common mode approach for the formulations of forward and inverse problems. To be precise, we split the total current vector potential $\mathbf{T}$ into a source field $\mathbf{H}_{\mathrm{s}}$ representing a common mode current density $\mathbf{J}_{0}=$ curl $\mathbf{H}_{\mathrm{s}}$ with its terminal current being equal to the prescribed total current $I_{\text {tot }}$ and into a reduced 
current vector potential $\mathbf{T}_{\mathrm{m}}$ representing the internal current loops $\mathbf{J}_{\mathrm{m}}=\operatorname{curl} \mathbf{T}_{\mathrm{m}}$. Then we have:

$$
\mathbf{J}=\nabla \times \mathbf{H}_{s}+\nabla \times \mathbf{T}_{m},
$$

where $\mathbf{T}_{\mathrm{m}}$ and $\mathbf{J}_{\mathrm{m}}$ are the unknowns in spaces $\mathrm{W}^{1}$ and $\mathrm{W}^{2}$, respectively. The current density can be written as the superposition of the common mode current density $\mathbf{J}_{0}$ plus an unknown current density $\mathbf{J}_{\mathrm{m}}$, i.e. $\mathbf{J}=\mathbf{J}_{0}+\mathbf{J}_{\mathrm{m}}$. Owing to the linear property of the Biot-Savart operator, we have:

$$
\left[B_{m}\right]=[B]-[L]\left[J_{0}\right]=[L]\left[J_{m}\right]
$$

Instead of solving for the total current density $\mathbf{J}$ in $\mathrm{W}^{2}$ or total current vector potential $\mathbf{T}$ in $\mathrm{W}^{1}$, we can solve for the reduced unknowns $\mathbf{J}_{\mathrm{m}}$ in $\mathrm{W}^{2}$ or $\mathbf{T}_{\mathrm{m}}$ in $\mathrm{W}^{1}$. Thus, we solve for $\left[J_{m}\right]$ from $\left[B_{m}\right]$ :

$$
\min _{\left[J_{m}\right]}\left\{\left\|[L]\left[J_{m}\right]-\left[B_{m}\right]\right\|_{2}^{2}+\lambda^{2}\left\|\left[J_{m}\right]\right\|_{2}^{2}\right\}
$$

or solve for $\left[T_{m}\right]$ from $\left[B_{m}\right]$ :

$$
\min _{\left[T_{m}\right]}\left\{\left\|[L][C]\left[T_{m}\right]-\left[B_{m}\right]\right\|_{2}^{2}+\lambda^{2}\left\|[K]\left[T_{m}\right]\right\|_{2}^{2}\right\} .
$$

The source field $\mathbf{H}_{\mathrm{s}}$ describes an arbitrary source current distribution $\mathbf{J}_{0}$ that is supposed to be known in $\Omega$ and must be zero outside $\Omega$. Moreover, $\mathbf{H}_{\mathrm{s}}$ should be tangentially continuous in the whole region, i.e. represented in $\mathrm{W}^{1}$. Possible choices for $\mathbf{H}_{\mathrm{s}}$ and $\mathbf{J}_{0}$ have been discussed in (Biro et al., 1993). In this work, we solve an electrokinetic problem by imposing a uniform conductivity distribution $\sigma_{c m}$ in arc chamber with $\sigma_{c m}<<\sigma_{e}$, where $\sigma_{e}$ is the conductivity of the electrodes. Then, we obtain $\left[J_{0}\right]$ as the vector of degree of freedoms for $\mathbf{J}_{0}$ by projecting $\mathbf{J}_{0}$ into space $\mathrm{W}^{2}$. $\left[H_{s}\right]$ can be calculated from $\left[J_{0}\right]$ by using Biot-Savart law.

\section{Results}

The proposed approaches are numerically tested with 256 simulated magnetic field values of a 16-by-16 sensor array. The magnetic sensors are assumed to be able to measure the normal component of the magnetic induction, i.e. $x$-component as in Figure 1. The model of the arc chamber shown in Figure 1 is discretized using tetrahedrons with $N=392, E=2,037, F=3,004$ and $V=1,358$. Taking into account the implementation of boundary conditions in space $\mathrm{W}^{2}$, we finally have 2,440 unknowns when solving for [J or $\left[J_{\mathrm{m}}\right]$. The condition number of the kernel matrix is $1.56 \times 10^{6}$. With the implementation of boundary conditions and the tree gauging condition, we have 1,646 unknowns when solving for $[T]$ or $\left[T_{\mathrm{m}}\right]$ in space $\mathrm{W}^{1}$ with the condition number as $6.88 \times 10^{5}$. That means, whether we solve the inverse problem in $\mathrm{W}^{2}$ or in $\mathrm{W}^{1}$, the inverse problem is strongly underdetermined and ill-conditioned. The singular value decomposition is used to calculate the Tikhonov regularized solution. The optimal regularization parameter is computed by the L-curve method using a MATLAB package of regularization tools (Hansen, 2007). 
To avoid the inverse crime, the reference current density $\left[J_{\text {ref }}\right]$ and the common mode current density $\left[J_{0}\right]$ and their corresponding magnetic fields $[B]$ and $\left[B_{0}\right]$ are obtained in DC with $\varphi-A$ formulation using a commercial FEM software (AC/DC module user's guide, $\mathrm{v}$. 5.3a, COMSOL, Inc, 2017). A non-uniform conductivity distribution $\sigma_{a r c}$ is imposed in the arc chamber to calculate $\left[J_{\text {ref }}\right]$ and its corresponding $[B]$. The distribution of $\left[J_{\text {ref }}\right]$ and $\left[J_{0}\right]$ are shown in Figure 2.

The reconstructions of current density by solving for $\mathbf{J}$ in $\mathrm{W}^{2}$ and by solving for $\mathbf{T}$ in $\mathrm{W}^{1}$ without the proposed common mode approach are presented in Figure 3. Large errors are found in the reconstructions as we can see from the pictures. The reconstructions can be significantly improved with the proposed common mode approach, i.e. solving for $\mathbf{J}_{\mathrm{m}}$ in $\mathrm{W}^{2}$ and $\mathbf{T}_{\mathrm{m}}$ in $\mathrm{W}^{1}$, as shown in Figures $4(\mathrm{a})$ and (c). Standard Tikhonov regularization, i.e. the regularization operator $[K]=[I]$, is used when solving the inverse problem in space $\mathrm{W}^{2}$. However, the standard Tikhonov regularization fails to obtain a useful result, as shown in Figure 4(b). Noting that the standard Tikhonov regularization minimizes the norm of [T], we choose the discrete curl operator $[C]$ as the regularization operator, i.e. $[K]=[C]$, to minimize $[J]$ instead of [T], which may be physically reasonable.

To guarantee that the divergence of reconstructed current density is zero, an additional constraint is used when solving the inverse problem in space $\mathrm{W}^{2}$. One of the most important
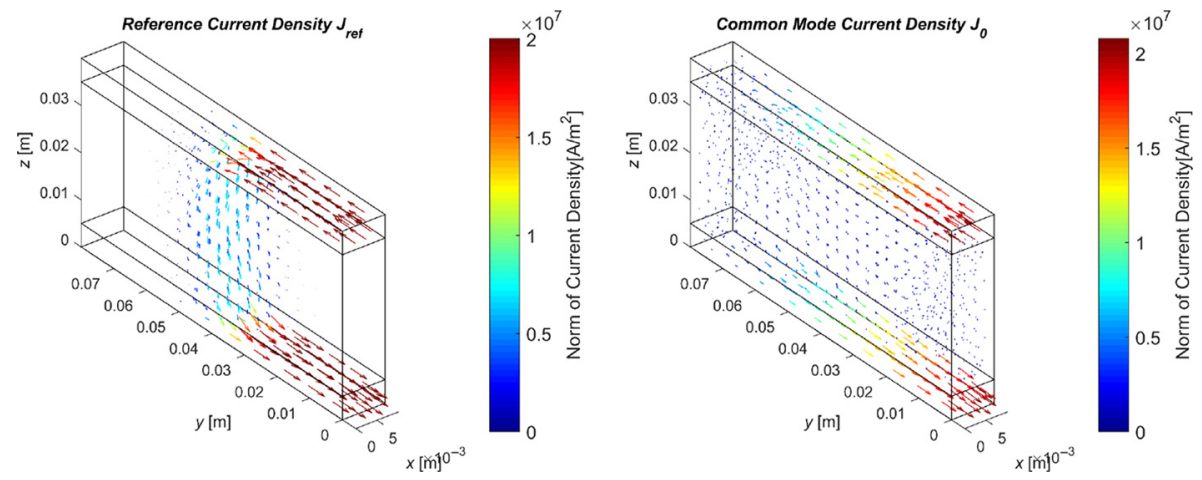

Figure 2.

Reference current density $\mathbf{J}_{\text {ref }}$ (left) and common mode current density $\mathbf{J}_{0}$ (right)
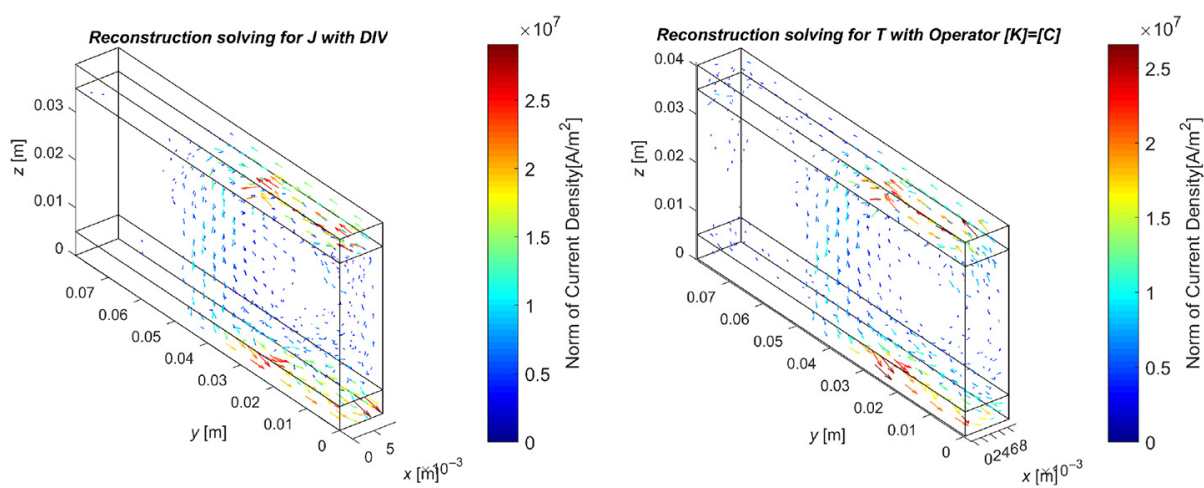

Figure 3.

Reconstructions of current density by solving for [J] in $\mathrm{W}^{2}$ (left) and by solving for $[\mathrm{T}]$ in $\mathrm{W}^{1}$ (right) 
COMPEL 38,3

Figure 4.

Reconstructions of current density

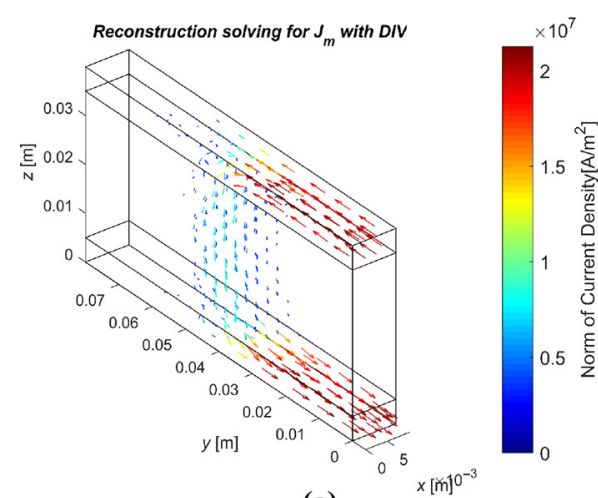

(a)

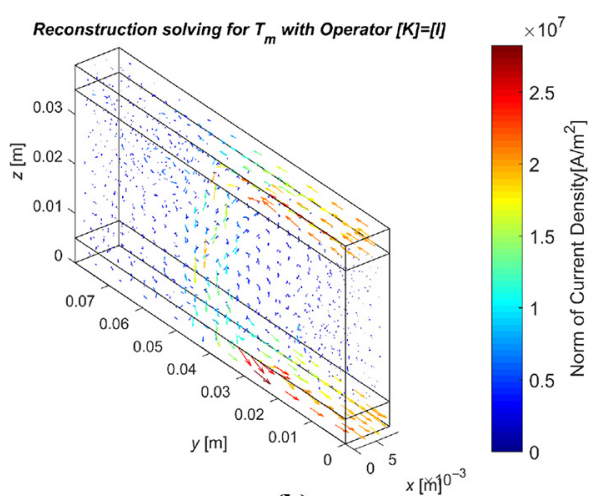

(b)

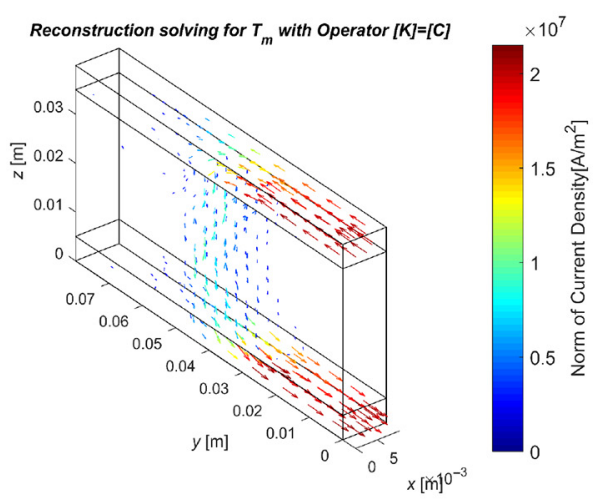

(c)

Notes: (a) By solving for $\left[\mathrm{J}_{\mathrm{m}}\right]$ in $\mathrm{W}^{2}$; (b) by solving for $\left[\mathrm{T}_{\mathrm{m}}\right]$ in $\mathrm{W}^{1}$ with the regularization operator $[\mathrm{K}]=[\mathrm{I}]$; (c) by solving for $\left[\mathrm{T}_{\mathrm{m}}\right]$ with $[\mathrm{K}]=[\mathrm{C}]$

benefits for solving the inverse problem in $\mathrm{W}^{1}$ using edge elements is that there is no need to implement the zero divergence condition. It can be noted that no big difference is found between the reconstructions in Figure 4(a) with zero divergence condition as a constraint and that in Figure 4(c), where the zero divergence condition naturally holds.

To analyze the effects of the mesh resolution on the arc current density reconstruction, a finer tetrahedral mesh is used with $N=1,313, E=7,735, F=12,220$ and $V=5,797$. The condition numbers of the kernel matrix when solving for $\mathbf{J}_{\mathrm{m}}$ and $\mathbf{T}_{\mathrm{m}}$ are $2.08 \times 10^{8}$ and $7.64 \times 10^{7}$, respectively. Figure 5 shows the improved reconstructions of the arc current density using the finer mesh.

From the comparison between the formulations using face elements with the one using edge elements, it can be noted that the use of edge elements requires a tree-cotree gauging to ensure a unique solution for the current vector potential. The choice of the tree affects significantly the accuracy and the use of an arbitrary tree may result in a poor convergence and accuracy or even in an unsolvable problem (Golias and Tsiboukis, 1994; Igarashi, 2001). The tree-cotree gauging has to search for an optimal minimum spanning tree, which can be difficult and time-consuming for complicated structures and finer meshes. Moreover, the 


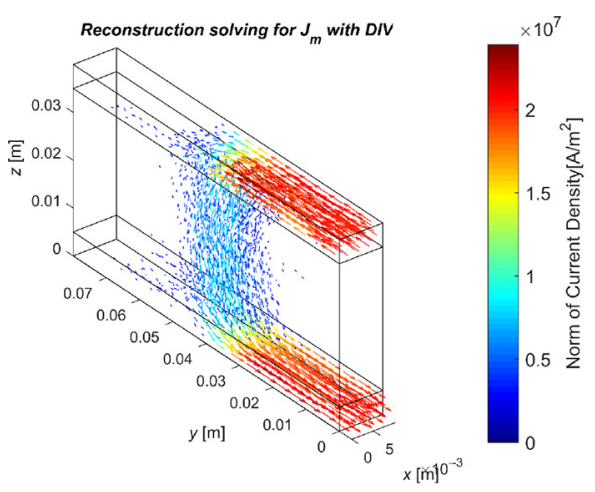

(a)

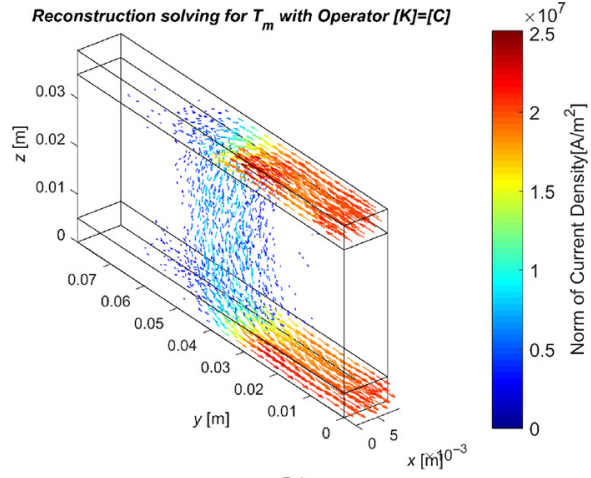

(b)

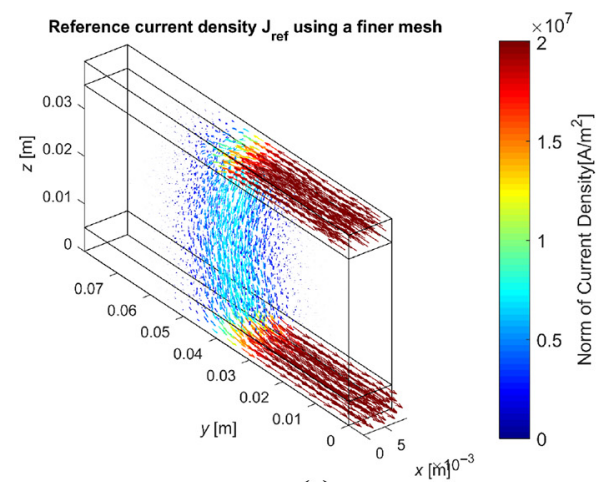

(c)

Figure 5.

Reconstructions with a finer mesh

Notes: (a) By solving for
reference current density

standard Tikhonov regularization (where the regularization operator is the identity operator) can obtain a good reconstruction when solving the inverse problem formulated using face elements, but fails in the case of edge elements. Thus, additional efforts have to be taken to find an optimal regularization operator for the inverse problem using edge elements. Given that both formulations can result in comparable accuracy in the reconstructions of the electric arc current density, the formulation using face elements in space $\mathrm{W}^{2}$ is to be preferred.

\section{Conclusion}

This paper presents a formulation of the magnetic inverse problem using Whitney elements to reconstruct the electric arc current density in a simplified arc chamber of low voltage circuit breakers. To guarantee that the divergence of the reconstructed current density is zero, two methods for the formulation of the inverse problem are proposed, i.e. the formulations using face elements in Whitney space $\mathrm{W}^{2}$ and using edge elements in Whitney space $\mathrm{W}^{1}$. An additional constraint is introduced using the discrete divergence operator 
when solving the inverse problem in $\mathrm{W}^{2}$, while zero divergence condition naturally holds when solving the inverse problem in $\mathrm{W}^{1}$. The proposed common mode approach has a key role to obtain a good reconstruction. No large difference are found between the reconstruction in $\mathrm{W}^{2}$ and the reconstruction in $\mathrm{W}^{1}$. In addition, when solving for $\mathrm{T}_{\mathrm{m}}$ in $\mathrm{W}^{1}$, the choice of the regularization operator has a very important influence on the reconstruction. From the numerical results, the discrete curl operator $[C]$ is a good option for the regularization operator, i.e. $[K]=[C]$, to ensure a stable and good solution. The formulation using face elements is to be preferred considering the complexity of the formulations.

In future work, we will consider more realistic geometries and magnetic field experimental data.

\section{References}

AC/DC module user's guide, v. 5.3a, COMSOL, Inc. (2017), available at: www.comsol.com

Bauer, M., Pursiainen, S., Vorwerk, J., Kostler, H. and Wolters, C.H. (2015), “Comparison study for Whitney (Raviart-Thomas)-type source models in finite-element-method-based EEG forward modeling", IEEE Transactions on Biomedical Engineering, Vol. 62 No. 11, pp. 2648-2656.

Biro, O., Preis, K., Renhart, W., Vrisk, G. and Richter, K.R. (1993), "Computation of 3-D current driven skin effect problems using a current vector potential”, IEEE Transactions on Magnetics, Vol. 29 No. 2, pp. 1325-1328.

Bossavit, A. (1988), "Whitney forms: a class of finite elements for three-dimensional computations in electromagnetism", Iee Proceedings A Physical Science, Measurement and Instrumentation, Management and Education, Reviews, Vol. 135 No. 8, pp. 493-500.

Brdys, C., Cajal, D., Toumazet, J.P., Gary, F., Laurent, A. and Arnoux, C. (2010), "Study of the arc behavior in low-voltage circuit breaker by means of optical and magnetic measurements", The European Physical Journal Applied Physics, Vol. 49 No. 2, 22904 (1-6).

Brdys, C., Toumazet, J.P., Laurent, A. and Ponthenier, J.L. (2002), "Optical and magnetic diagnostics of the electric arc dynamics in a low voltage circuit breaker", Measurement Science and Technology, Vol. 13 No. 7, pp. 1146-1153.

Calvetti, D., Reichel, L. and Shuibi, A. (2004), "L-curve and curvature bounds for Tikhonov regularization", Numerical Algorithms, Vol. 35 Nos 2/4, pp. 301-314.

Chadebec, O., Meunier, G., Mazauric, V.G., Le Floch, Y. and Labie, P. (2004), "Eddy-current effects in circuit breakers during arc displacement phase", IEEE Transactions on Magnetics, Vol. 40 No. 2 , pp. 1358-1361.

Di Rienzo, L., Haueisen, J. and Arturi, C.M. (2005), "Three component magnetic field data: impact on minimum norm solutions", Compel - the International Journal for Computation and Mathematics in Electrical and Electronic Engineering", Vol. 24 No. 3, pp. 869-881.

Dong, J., Zhang, G., Geng, Y. and Wang, J. (2018), "Current distribution reconstruction in low-voltage circuit breakers based on magnetic inverse problem solution considering ferromagnetic splitters", IEEE Transactions on Magnetics, Vol. 54 No. 10, 8001509(1-9).

Dong, J., Zhang, G., Geng, Y. and Wang, J. (2018), "Influence of magnetic measurement modelling on the solution of magnetostatic inverse problems applied to current distribution reconstruction in switching air arcs", IEEE Transactions on Magnetics, Vol. 54 No. 3, 8000704(1-4).

Ghezzi, L., Piva, D. and Di Rienzo, L. (2012), "Current density reconstruction in vacuum arcs by inverting magnetic field data", IEEE Transactions on Magnetics, Vol. 48 No. 8, pp. 2324-2333.

Golias, N.A. and Tsiboukis, T.D. (1994), "Magnetostatics with edge elements: a numerical investigation in the choice of the tree", IEEE Transactions on Magnetics, Vol. 30 No. 5, pp. 2877-2880. 
Hansen, P.C. (2007), "Regularization tools version 4.0 for matlab 7.3", Numerical Algorithms, Vol. 46 No. 2, pp. 189-194.

Hasani Eskandari, J. and Zareamoghaddam, H. (2014), "Different tikhonov regularization operators for elementary residual method", The SIJ Transactions on Computer Science Engineering and Its Applications (CSEA), Vol. 2 No. 1, pp. 19-24.

Hong, D., Sandolache, G., Bauchire, J.M., Gentils, F. and Fleurier, C. (2005), “A new optical technique for investigations of low-voltage circuit breakers", IEEE Transactions on Plasma Science, Vol. 33 No. 2, pp. 976-981.

Igarashi, H. (2001), "On the property of the curl-curl matrix in finite element analysis with edge elements", IEEE Transactions on Magnetics, Vol. 37 No. 5, pp. 3129-3132.

Lindmayer, M., Marzahn, E., Mutzke, A., Ruther, T. and Springstubbe, M. (2006), "The process of arc splitting between metal plates in low voltage arc chutes", IEEE Transactions on Components and Packaging Technologies, Vol. 29 No. 2, pp. 310-317.

McBride, J.W., Balestrero, A., Ghezzi, L., Tribulato, G. and Cross, K.J. (2010), "Optical fiber imaging for high speed plasma motion diagnostics: applied to low voltage circuit breakers", Review of Scientific Instruments, Vol. 81 No. 5, 055109 (1-6).

Meunier, G. (2008), “Chap. 2.3”, The Finite Element Method for Electromagnetic Modeling, Wiley, New York, NY.

Pursiainen, S., Sorrentino, A., Campi, C. and Piana, M. (2011), "Forward simulation and inverse dipole localization with the lowest order Raviart-Thomas elements for electroencephalography", Inverse Problems, Vol. 27 No. 4, 045003(1-17).

Shea, J.J. (2004), "Gassing arc chamber wall material effect on post current-zero recovery voltage breakdown", IEEE Transactions on Components and Packaging Technologies, Vol. 27 No. 1, pp. $42-50$.

Tanzer, I.O., Jarvenpaa, S., Nenonen, J. and Somersalo, E. (2005), "Representation of bioelectric current sources using whitney elements in the finite element method", Physics in Medicine and Biology, Vol. 50 No. 13 , pp. 3023-3039.

Toumazet, J.P., Brdys, C., Laurent, A. and Ponthenier, J.L. (2005), "Combined use of an inverse method and a voltage measurement: estimation of the arc column volume and its variations", Measurement Science and Technology, Vol. 16 No. 7, pp. 1525-1533.

Wu, Y., Rong, M., Li, J. and Lou, J. (2006), "Calculation of electric and magnetic fields in simplified chambers of low-voltage circuit breakers", IEEE Transactions on Magnetics, Vol. 42 No. 4, pp. 1007-1010.

Yang, F., et. al. (2013), "Low-voltage circuit breaker arcs - simulation and measurements", Journal of Physics D: Applied Physics, Vol. 46 No. 27, p. 273001.

\section{Corresponding author}

Luca Di Rienzo can be contacted at: luca.dirienzo@polimi.it 